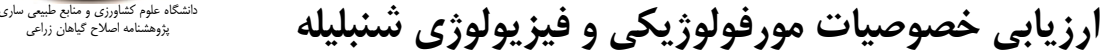

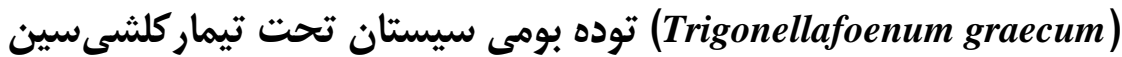

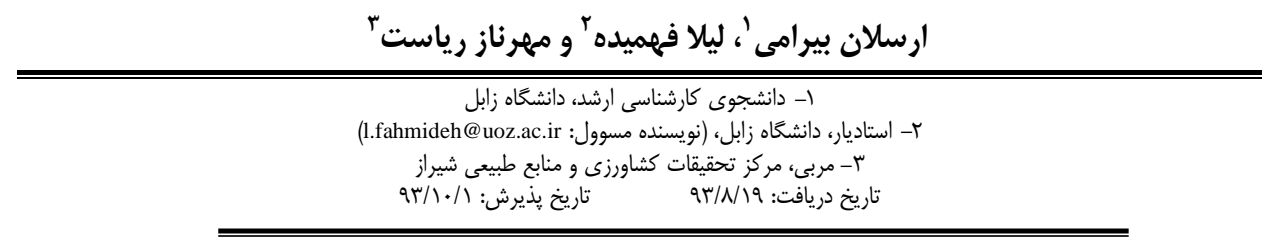

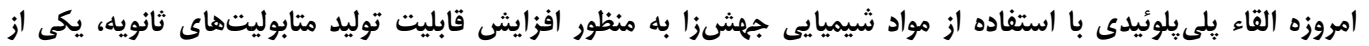

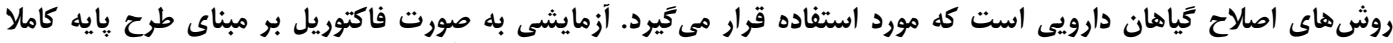

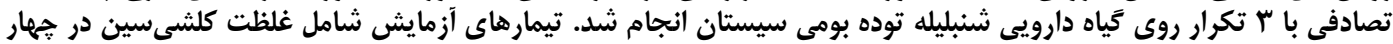

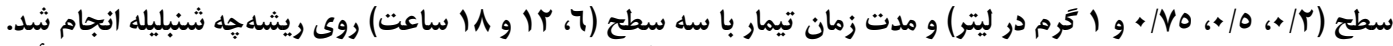

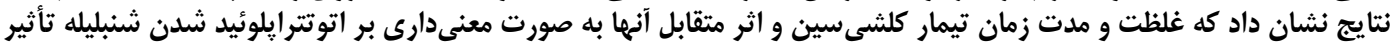

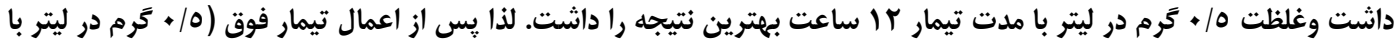

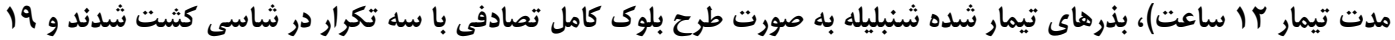

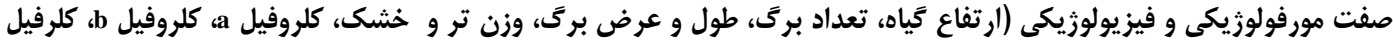

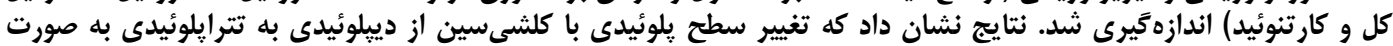

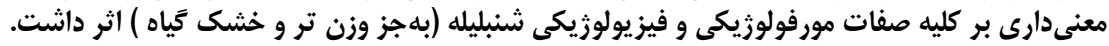

وازههاى كليدى: شنبليله، كلشىسين، اتوتترايلوئيدى، خصوصيات مورفولوزيك و فيزيولوزيك

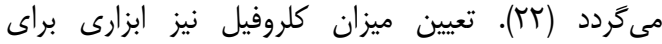

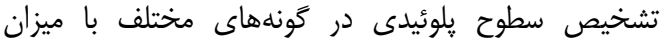

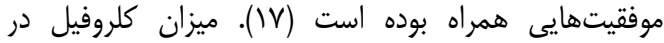

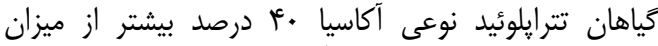

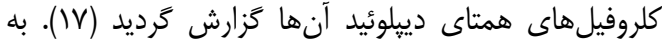

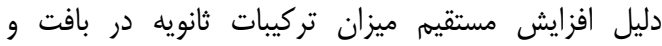

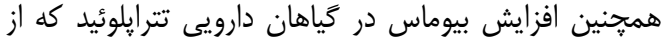

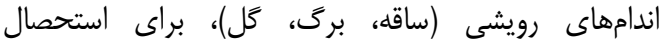

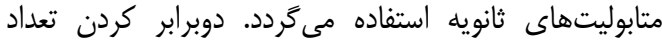

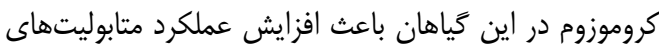

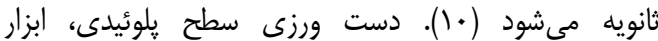

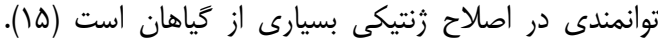

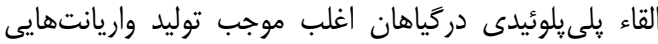

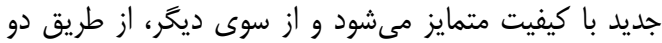

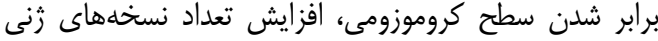

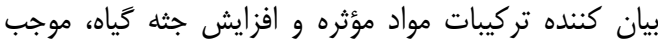

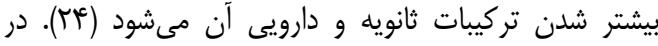

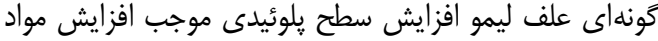

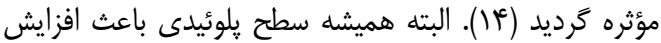

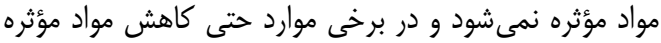

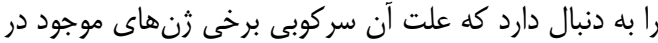

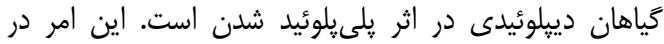

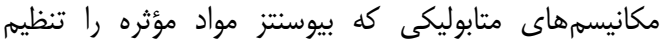

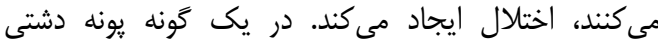
(Menthaarvensis) افزايش اسانس داشته ولى دركّونه (M.spicata) ميزان دان درائل
شنبليله يك كياه شناخته شده و معروف است كه اثرات

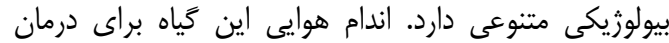

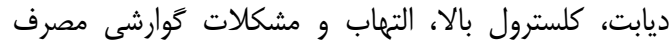

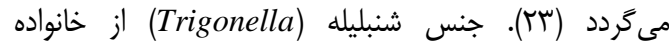

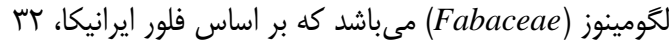

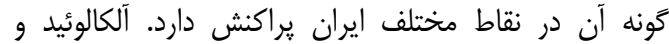

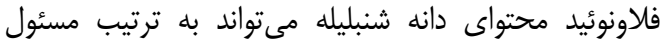

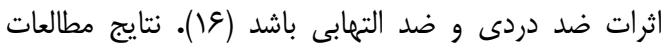

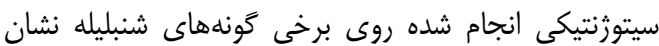

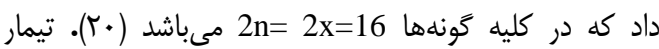

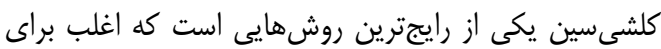

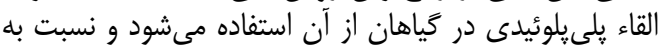

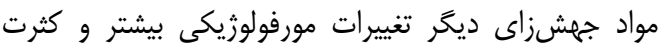

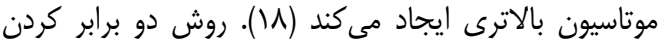

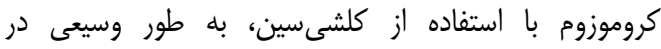

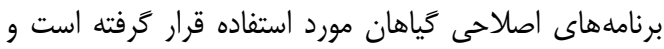

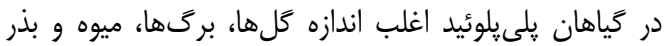

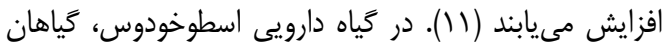

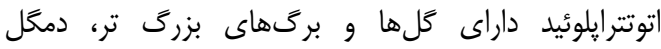

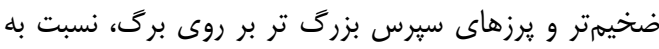

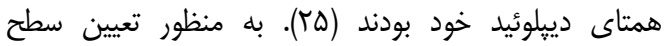

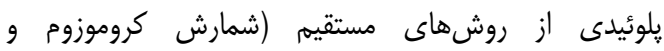

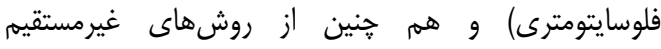

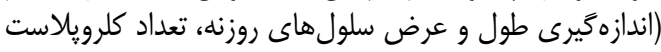
سلولهاى محافظ روزنه و مشاهدات مورفولوزيكى) استفاده 
جلو إيرى از نفوذ هوا در زير لامل، يوششى از لاكى در اطراف

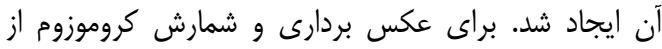

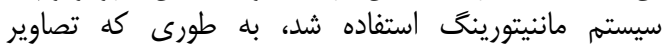

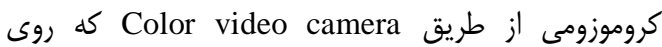
ميكروسكوٍ نورى نصى نصب شده بود به مانيتور منتقل شده و ضبط ترديد.

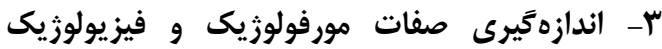
كَياهان تترايلوئيد و دييلوئيد

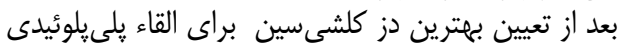

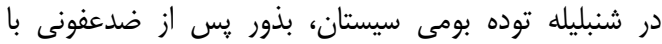

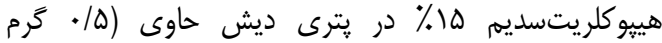

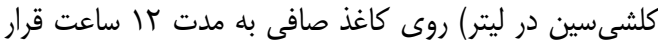

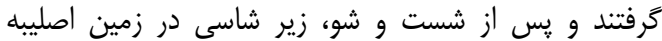

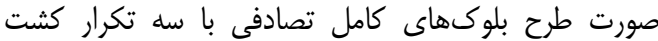

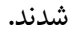

يس از كشت بذرها برخى صفات مورفولوزيكى و و

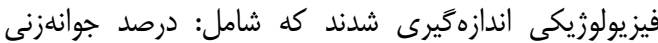

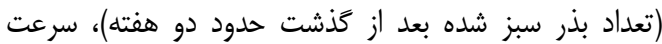

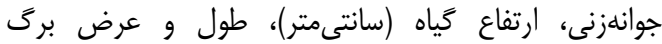

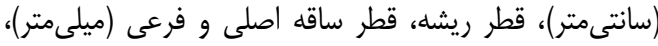
تعداد شاخه جانبى، تعداد برى رئ، تعداد

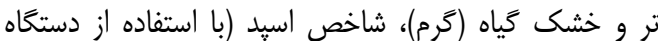

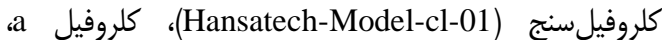
كلروفيل bا كلروفيل كل و كارتنوئيد بودند. دادههاى بلهدست

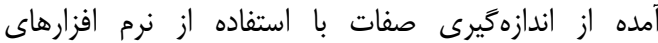

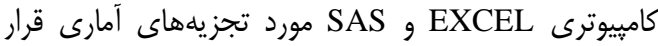

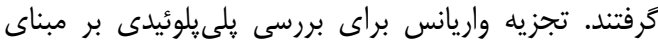

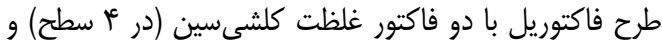

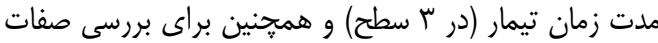

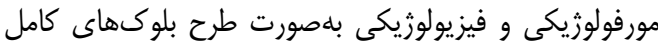

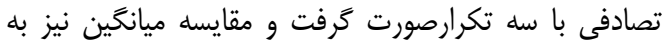
روش دانكن انجام گرديد.

\section{نتايج و بحث}

يس از مطالعه و عكسبردارى از از اسلايد نمونه شاهد، عدد

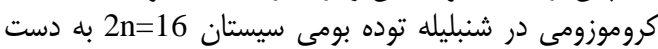

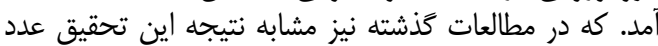
كروموزومى گايايه در شنبليله

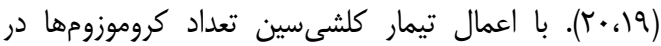

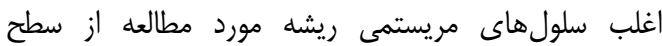

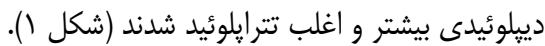

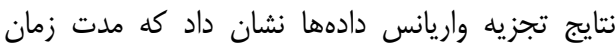

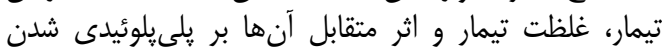

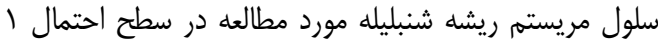

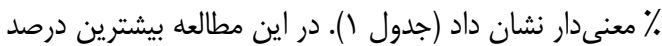

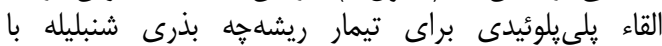

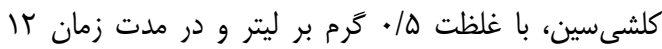

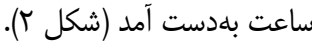

اسانس كاهش يافته است. همجنين در كل انگشتانه ارغوانى،

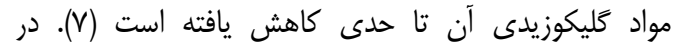

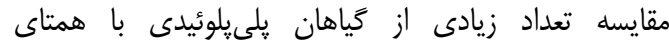

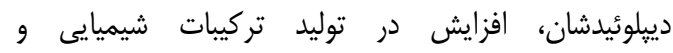

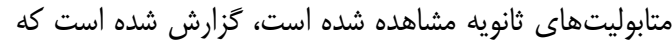

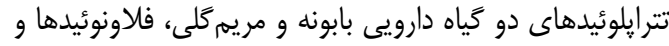

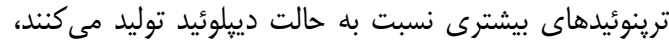

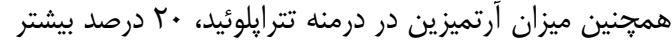

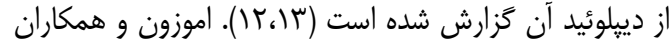

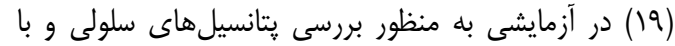

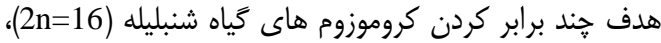

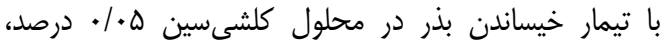

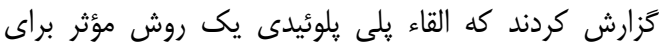

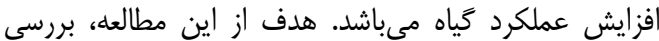

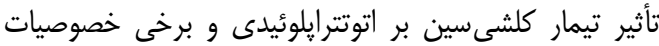

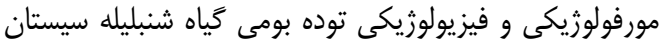
مىباشد.

مواد و روشها

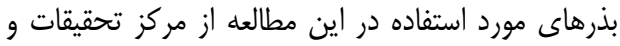

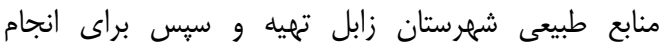

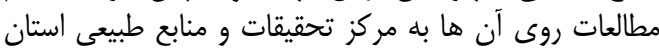

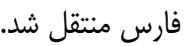

اـ كثت و وآماده سازى بذر بذرها

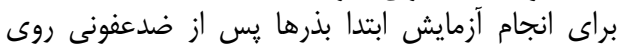

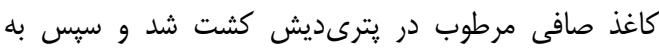

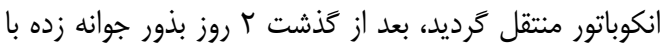

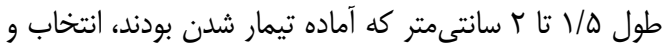

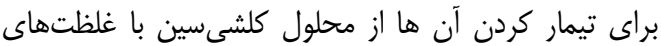

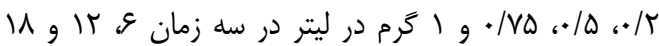
ساعت استفاده شد. r- شمارش كروموزومها به بـ روش اسكواش

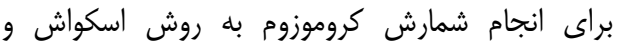

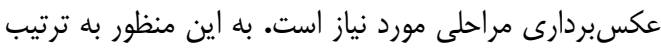

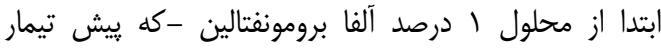

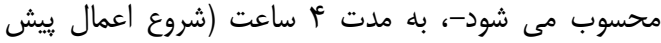

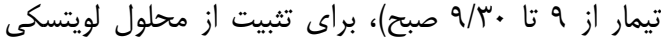

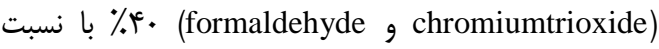

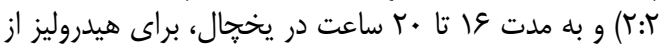

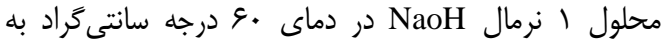

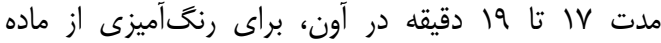

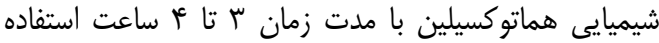

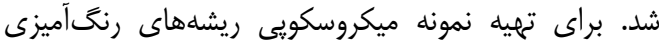

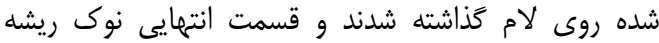

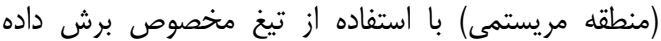

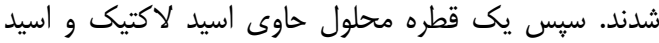

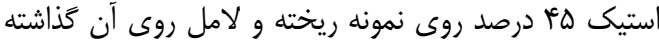

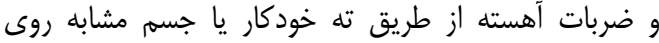
لامل وارد شد (به طورى كه لامى حركت نكند) و بر براى 


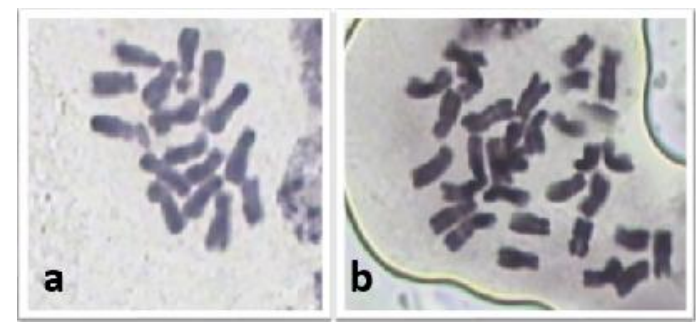

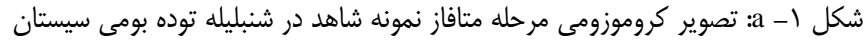

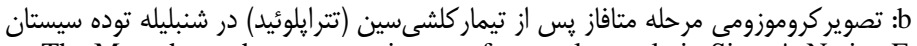

Figure 1. a. The Metaphase chromosome image of control sample in Sistan's Native Fenugreek b: Metaphase chromosome image after treating with colchicine (tetra ploid) in Sistan's Native Fenugreek

جدول ا- نتايج تجزيه واريانس القاء يلىيلوئيدى يس از تيمار با كلشىسين

Table 1. The results of analysis of variance for polyploidy induction after treating with colchicine

\begin{tabular}{|c|c|c|}
\hline & ميانكين مربعات & \\
\hline يلي يلوئيدى (\%) & ل درجه آزادى & منابع تغييرات \\
\hline $1 \cdot \mu \mathrm{M} / \mathrm{AV}$ & $r$ & زمان \\
\hline TYVT/MY & r & غلظت \\
\hline TrE/AV & 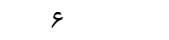 & زمان×غلظت \\
\hline $1 / v$ & $\Delta \Lambda$ & خطا \\
\hline$r / \mathrm{VV}$ & & ضريب تغييرات (.٪) \\
\hline
\end{tabular}

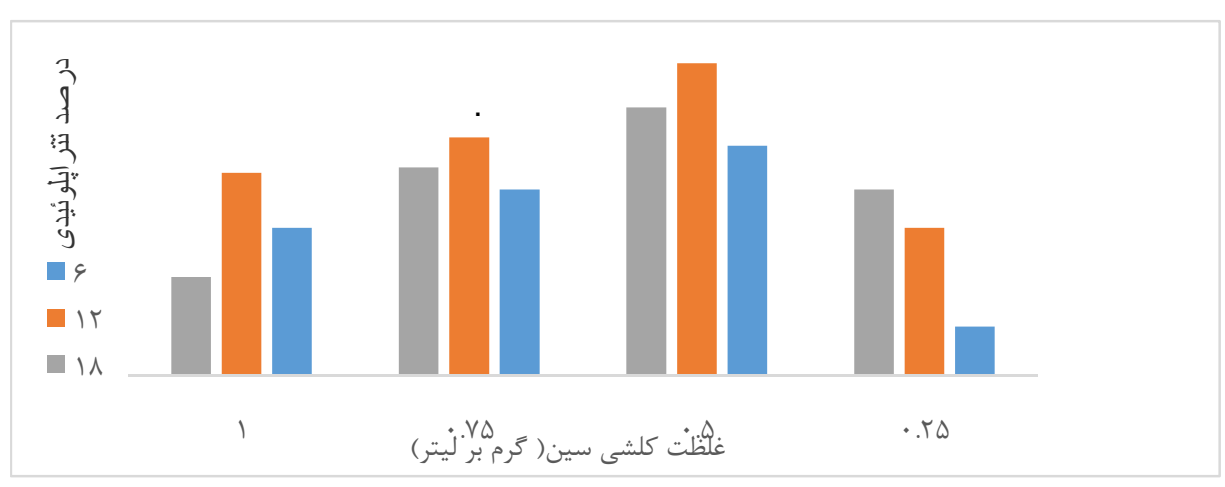

شكل r- اثر متقابل غلظت و ملت زمان تيمار كلشىسين

Figure 2. Interaction effect of concentration and period of colchicines' treatment

همجنين طبق كزارش اسماعيلحسنى و همكاران (9) كياهان

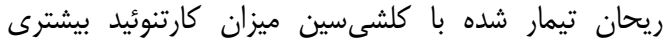

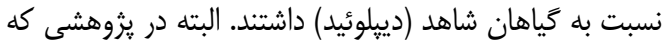

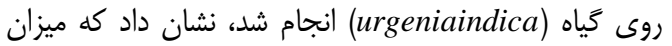

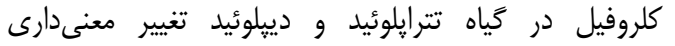

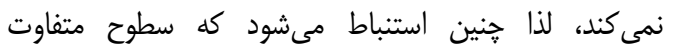

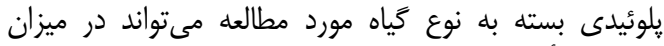
كلروفيل تأثير داشته باشد (ه).
كلروفيل و كار تنوئيد

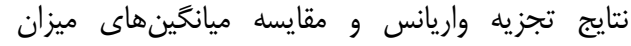

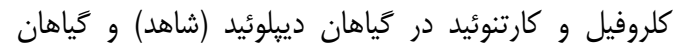

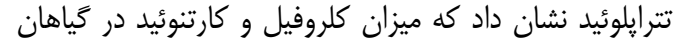

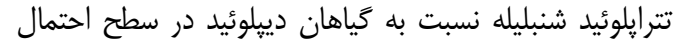

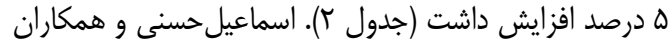

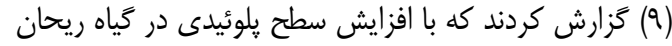

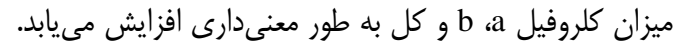

جدول r- نتايج تجزيه واريانس صفات فيزيولوزيكى پيس از تيمار با كلشىسين

Table 2. The results of analysis of variance for physiologic traits after treating with colchicine

\begin{tabular}{|c|c|c|c|c|c|}
\hline & & يانكين مربعات & & & \\
\hline كاتنوئيد & كلروفيل كل & bلروفيل bل b & a كلروفيلa & درجه آزادى & منابع تغييرات \\
\hline$\cdot / \cdots \cdots \cdot \Lambda^{\text {nI }}$ & $\cdot / \cdot r F \Delta^{\text {IIS }}$ & $\cdot / \cdot r r \lambda^{115}$ & $\cdot / \cdots \cdot 10^{115}$ & $r$ & تكرار \\
\hline.$/ \ldots q g r^{*}$ & $.1999 . .^{*}$ & $\cdot / r \cdot r \Delta \cdot *$ & . & 1 & تيمار \\
\hline $.1 .+r F$ &.$/ . r 190$ & ...FAT & |... | & r & خطا كل \\
\hline$r / 川$ & $4 / \cdot 1$ & $\Delta / 8 \Lambda$ & $r / r$. & & ضريب تغييرات \\
\hline
\end{tabular}


Table 3. Mean comparison of physiologic traits

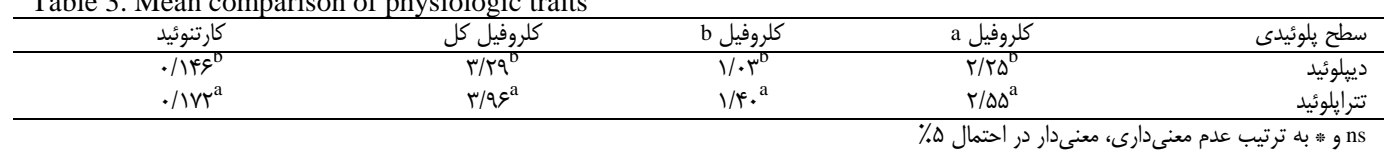

مقايسه ويثَى هاى كمى از قبيل: ارتفاع، طول بركى، انشعاب

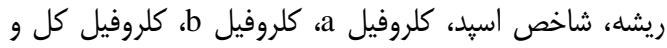

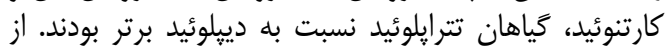

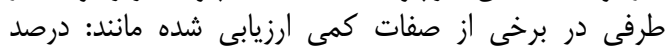

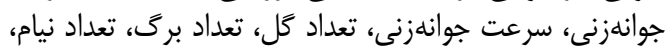

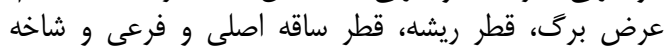

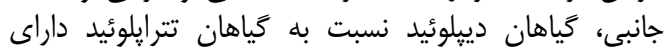

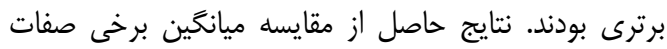

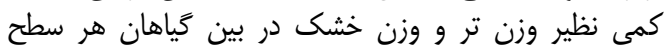

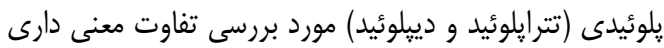

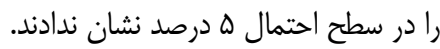

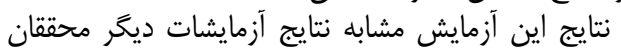

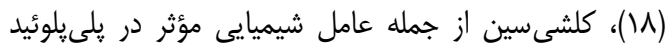

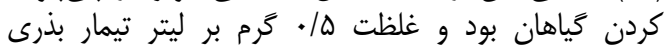

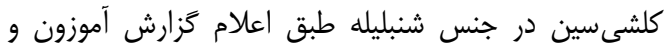

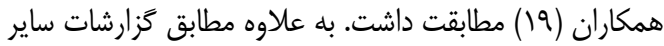

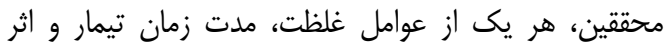

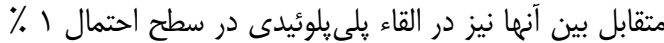

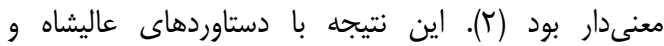

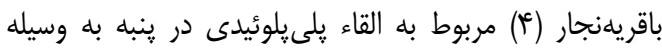

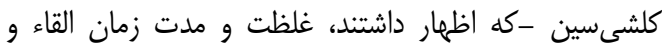

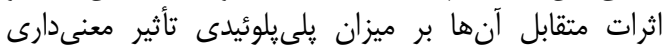

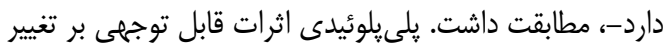

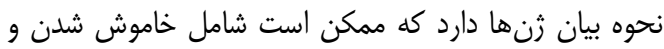

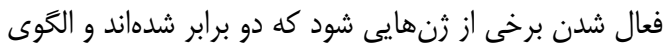

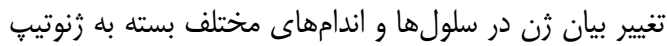

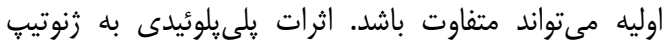

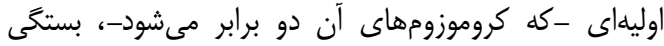

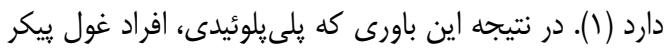

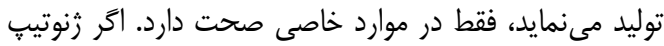
خيلى هتروزايخوس باشد، مانند (Oenotheralamarkiana)

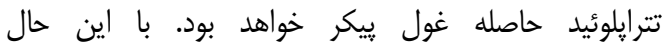

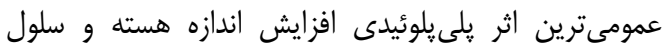

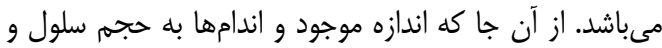

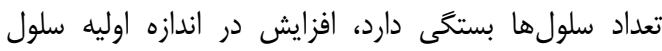

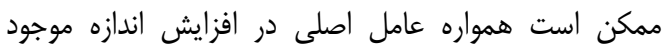

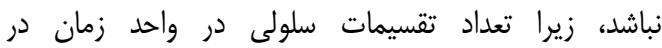

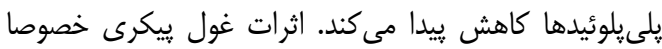

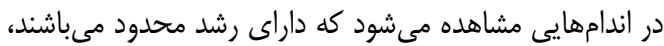

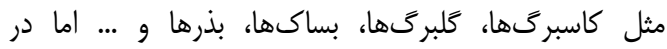

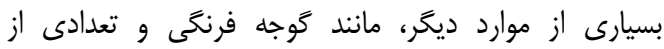

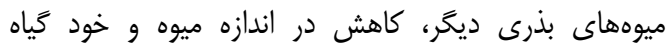

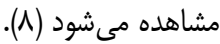

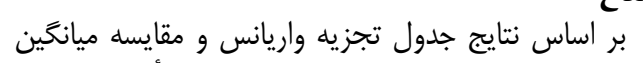
ار تفاع

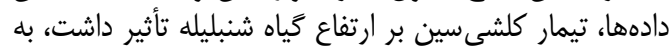

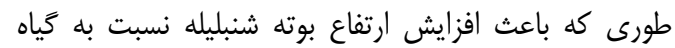

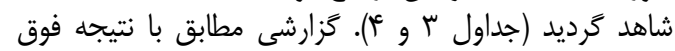

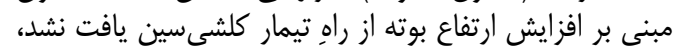

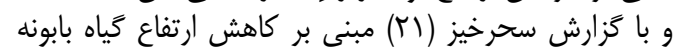
كبير با افزايش غلظت كرخي كلشىسين مغاير بود.

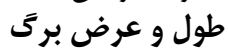

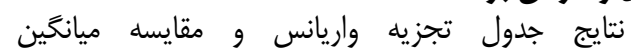

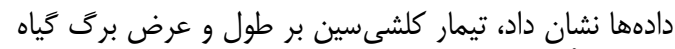

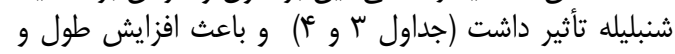

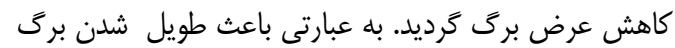

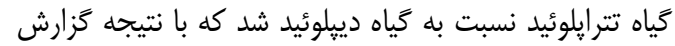

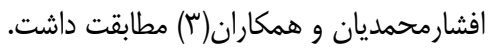

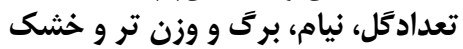

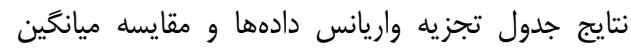

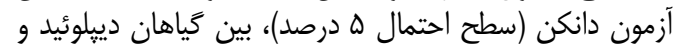

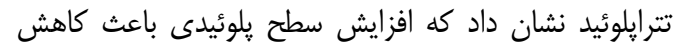

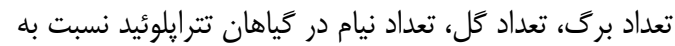

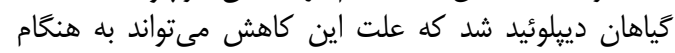

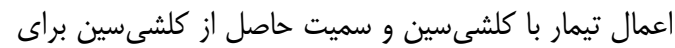

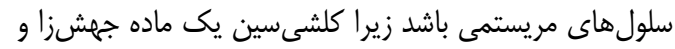

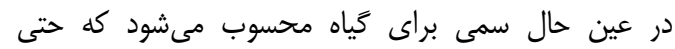

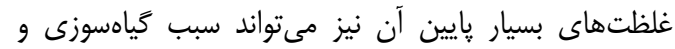

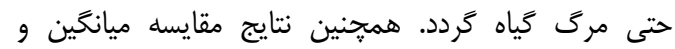

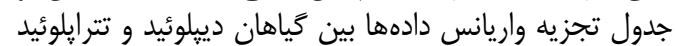

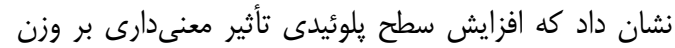

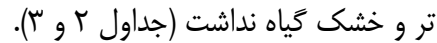
درصد جوانهزنى، سرعت جواهن (جرانهزنى، قطر ريشه و شاخص اسيد با توجه به نتايج مقايسه ميانكين در اين تحقيق مشاهده

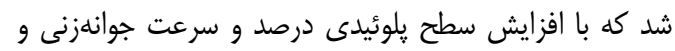

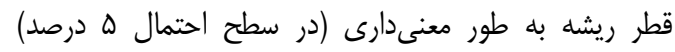

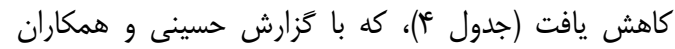

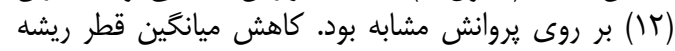

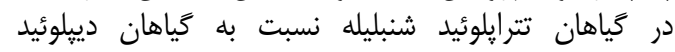

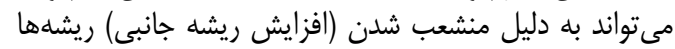

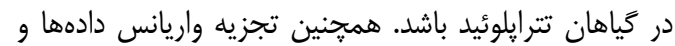

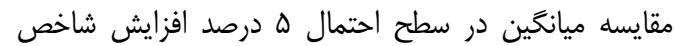

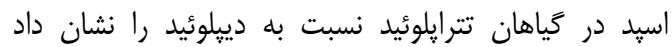

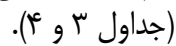

به طور كلى نتايج اين تحقيق نشان داد كه در بررسى و 


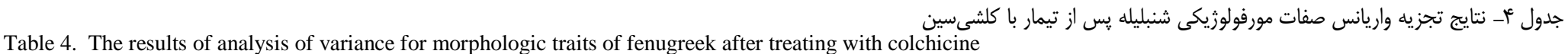

\begin{tabular}{|c|c|c|c|c|c|c|c|c|c|c|c|c|c|c|c|c|}
\hline شاخص & جوانةزعتى & جوانةزنى & خشك & وزن تر & قطر ريشه & تعداد بركى & تدر بوته & 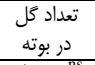 & فرعى ساقه & قطر ساقه & شانبى & برى & طول بركى & ارتفاع & آزادى & منابع تغييرات \\
\hline$\cdot\left(\cdot \Delta \mid f^{\mathrm{nS}}\right.$ & . (VY) & $\Gamma /\left.\varphi Q\right|^{\mathrm{nS}}$ & $\begin{array}{c}r M \backslash\rangle^{\text {nS }} \\
. /\end{array}$ & $r / T V \Delta .^{n S}$ & $\cdot / \cdot 10^{n t s}$ & $\begin{array}{c}10 \ldots{ }^{\mathrm{nS}} \\
r \wedge\end{array}$ & $\cdot / \Delta \cdot \cdot^{\mathrm{ns}}$ & $\cdot / 1999^{\mathrm{nI}}$ & $\cdot / \cdots \gamma^{\text {ns }}$ &.$/ 1199^{n s}$ & $1 / / 999^{\mathrm{nS}}$ & $\cdot / \cdot r q^{n s}$ & $\cdot / \cdot \wedge \mathrm{V}^{\mathrm{ns}}$ & $r / T V \Delta \theta^{n S}$ & $r$ & تكرار \\
\hline 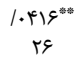 & $1 \% / \Delta \cdot .^{* *}$ & 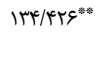 & $\begin{array}{c}M 1 . \mathrm{n}^{\mathrm{ns}} \\
\cdot /\end{array}$ & $N / 99^{\mathrm{ns}}$ & $1 / \Delta . .{ }^{a *}$ & $\begin{array}{l}198^{\circ 0} \\
94.5\end{array}$ & $r \cdot / 199^{* * *}$ & $r V / \Delta \cdot *^{*}$ & $1 / 451 . * *$ & r/q9A." & $\mid / 1999^{\circ}$ & 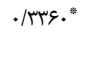 & $\cdot \mid r\left(\left.\Lambda \Lambda\right|^{*}\right.$ & $|\xi| \cdot+\mid q^{*}$ & 1 & تيمار \\
\hline /.rI9 & $\%+\varphi \Delta$. & $r / \Delta \cdot 1$ & $\begin{array}{c}1 \cdot v 19 \\
.\end{array}$ & $1 / 1999$ & $\% \Delta$ & r/1999 & .1999 & $\cdot / \Delta \cdot$. & $\%$ $/ \cdots$ & . &.$/ 1999$ & $\cdot / \cdot A V$ & $\% \Delta \Delta$ & . & r & خطا كل \\
\hline$T / T \Lambda$ & $F / V^{k}$ & $1 / 99$ & IV/SD & $\mid \pi / \mu \Lambda$ & $T / \mu^{2}$ &.$/ 91$ & $r / \Lambda$. & $V / V I$ & $\cdot / \Lambda \mu$ & $11 / \% 4$ & $1 . / 9)^{2}$ & $\Delta / r q$ & $T / F T^{\prime}$ & $1 / \pi \Delta$ & & ضريب تغييرات \\
\hline
\end{tabular}

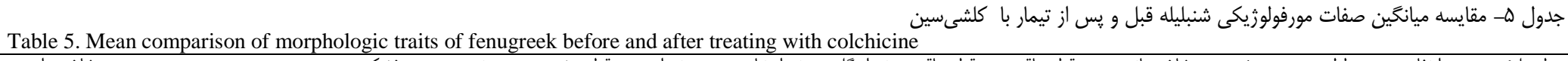

\begin{tabular}{|c|c|c|c|c|c|c|c|c|c|c|c|c|c|c|c|}
\hline شاخص اسيد & جوانهزنى & جوانهزنى & وزن خشك & وزن تر & قطر ريشه & تعرى & تعداد نيام در & تعد بوته & فرعى ساقه & قطر ساقه & شاخه جانبى & برضى عرض & بركَ & ارتفاع & سطح يلوئيدى \\
\hline$r / \mathrm{rq}^{0}$ & $\Delta / q^{a}$ & $99 / \lambda^{a}$ & $1 / 90^{a}$ & $11 / 19 v^{a}$ & $r / \kappa^{a}$ & $|1| / q^{a}$ & $1 . / r^{r^{a}}$ & $11 / \varepsilon q^{a}$ & $r / r r^{a}$ & $r / \Delta r^{a}$ & $\varphi / 9 q^{a}$ & $1 / 90^{a}$ & $r / Q \Lambda^{0}$ & $r V / \& q^{\circ}$ & دييلوئيد \\
\hline$s \mid \Delta \Lambda^{\mathrm{a}}$ & $r / q V^{D}$ & $9 . / \mu \Gamma^{0}$ & $1 / 1^{\mathrm{a}}$ & $N / \wedge r^{\mathrm{a}}$ & $r / \mathscr{F}^{\mathrm{D}}$ & $\| \varepsilon / \Gamma^{0}$ & $9 / 98^{\circ}$ & $9 / 98^{\circ}$ & $1 / T V^{D}$ & $r / I^{D}$ & $r^{\mathrm{D}}$ & $1 / \& \Psi^{D}$ & 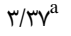 & $r \mid / \wedge r^{a}$ & تترايلوئيد \\
\hline
\end{tabular}


1. Adams, K.L and J.F. Wendel. 2005. Polyploidy and genome evolution in plants. CurrOpin Plan Biology, 8: 135-141.

2. Afshari, E., GH. Ranjbar, K. kazemitabar, M. riasat and H. kazemiposhtmasari. 2013. Effect of colchicine and trifluralin on root meristematic cells and cytogenetic characteristics of fenugreek (Trigonella foenum_graecum). Iranian Journal of Medicinal and Aromatic Plants Research, 29: 936951 (In Persian).

3. Afsharmohammadian, M., R. por akbarikamsaii, Z. omidi, F. ghanati and A. torang. 2012. Morphological and physiological effects induced polyploidy in plant lemon. Journal of Plant Biology, 4: 13-24 (In Persian).

4. Alishah, O. and M. Baghrrieh_najjar. 2008. Poly pioidization effect in two diploid cotton (Gossypium herbaceum and G. arboretum) species by colchicine treatments. African Journal of Biotechnology, 7: $102-108$.

5. Andersson, S.C. 2009. Carotenoids tocochromanols and chlorophylls in sea buckthorn berries (Hippophaerhamnoides) and rose hips (Rosa sp.). Ph.D. Thesis, Swedish University of Agricultural Sciences Alnarp, Sweden, 128 pp.

6. Behradmanesh, M., M. ahmadi and M. rafiyankopayi. 2012. Investigate the effect of glucose tablets Glycogol on type 2 diabetes. Iranian Journal of Endocrinology and Metabolism. Martyr Beheshti University of Medical Sciences and Health Services. 2: 163-168 (In Persian).

7. Dhawan, O.P. and U.C. Lavania. 1996. Enhancing the productivity of secondary metabolites via induced polyploidy. A review Euphytica, 87: 81-89.

8. Ehdayi, B. 1994. Plant Breeding.Tehran University Press, 589 pp (In Persian).

9. Esmailhasani, M., M. mirzaii, R. omidbeygi and M. fathigharehbaba. 2010. Autotetraploidy Effect on quantitative and qualitative properties of essential oils and herb basil. Iranian Journal of Horticultural Science, 41: 111-118 (In Persian).

10. Gonzalez, L.D.J. and P.J. Weathers. 2003. Tetraploid (Artemisia annua) hairy roots produce more artemisinin than diploids. Plant Cell Reports, 21: 809-811.

11. Hartwell, L.H., L. Hood, M.L. Goldberg, A. E. Reynolds, L.M. Silver and R.C. Veres. 2004. Genetics from genes to genomes, (2nd ed.). McGraw Hill, Boston, 324 pp.

12. Hoseini, H, M. chehrazi, D. nabatiahmadi and M. mahmodisarvestani. 2012. Induction PolyPloidy in Vinca plants and changes in phenotypic properties. First National Conference Playbooks achieving sustainable development, 5 pp (In Persian).

13. Jesus, L.D. 2003. Effect of artificial poly ploidy in transformed roots of (Artemisia annua L.) Plant Cell Reports, 21: 809-813.

14. Lavania, U.C. 1998. Enhanced productivity of the essential oil in the artificalautotertraploid of Vetiver (Vetiveria zizanioides L. Nash).Euphytica, 38: 271-276.

15. Madon, M., M.M. Clyde, H. Hashim, Y. Mohdyusuf, H. Mat and S. Saratha. 2005. Polyploidy induction of oil palm through Colchicine and oryzalin treatments. Journal of Oil Palm Research, 17: 110-123.

16. Mandegari, A., M. Pournamdari, F. Sharififar, Sh. Pournourmohammadi, R. Fardiar and S. Sholi. 2012. Alkaloid and flavonoid rich fractions of fenugreek seeds (Trigonellafoenum-graecum L.) with antinociceptive and anti- inflammatory effects. Food and Chemical Toxicology, 50: 2503-2507.

17. Mathura, S., A. Fossey and S. Beck. 2006. Comperative study of chlorophyll content in diploid and tetraploid black Wattle (Acacia mearnsii). Journal Forestry, 79: 381-388.

18. Mensha, J.K., B.O. Obadoni, P.A. Akomeah, B. Ikhajiagbe and J. Ajibolu. 2007. The effects of sodium azide and colchicine treatments on morphological and yield traits of sesame seed (Sesame indicum L.). African Journal of Biotechnology, 6: 534-538.

19. Omezzin, F., A. Ladhari, F. Nefzi, R. Harrath, M. Aouni and R. Haouala. 2012. Induction and flow cytometry identification of mixoploidy through colchicine treatment of (Trigonellafoenum graecum L.).African Journal of Biotechnology, 11: 16434-16442.

20. Riasat, M., Zh. Karaptyan and A. nasirzadeh. 2003. Study of karyotype of some species of the genus Trigonella fars Province. Iranian Journal of Rangelands and Forests Plant Breeding and Genetic Research. 11: 127-145 (In Persian).

21. Saharkhiz, M.J. 2006. Effect of climatic factors and ploidy level on morphological and physiological characteristics and ornamental herb chamomile Great. PhD thesis, Tarbiat Modarres University, Tehran. 173 pp (In Persian).

22. Sari, N., K. Abak and M. Pitrat. 1999. Comparison of ploidy level screening methods in Watermelon.ScientiaHorticulturae, 82: 265-277.

23. Sharma, R.D., A. Sarkar, D.K. Hazra, B. Mishra, J.B. Singh, S.K. Sharma, B.B. Maheshwari and P.K. Maheshwari. 1996. Use of fenugreek seed powder in the management of noninsulin dependent diabetes mellitus. Nutrition Research, 16: 1331-1339.

24. Thao, N.T.P., K. Ureshino, I. Miyajima, Y. Ozaki and H. Okubo. 2003. Induction of tetraploids in ornamental Alocasia through colchicine and oryzalin treatments. Plant cell, Tissue and Organ Culture, 72: $19-25$.

25. Urwin, N.A.R., J. Horsnell and T. Moon. 2007. Generation and characterization of colchicine-induced autotetraploid. (Lavandula angustifolia). Euphytica, 156: 257-266. 


\title{
Evaluation of Morphologic and Physiologic Traits of Sistan's Native Fenugreek (Trigonellafoenum graecum) under Colchicine Treatments
}

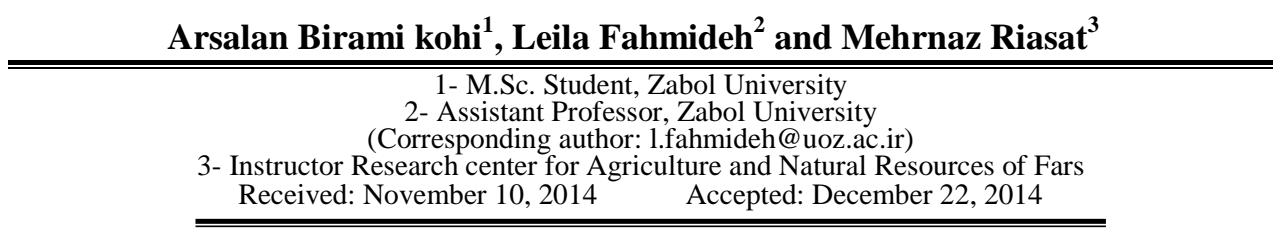

\begin{abstract}
Nowadays induction of polyploidy using mutagenic chemicals to increase secondary metabolic production as a modification method of pharmaceutical plants is very usual. A factorial experiment based on completely randomized design with three replications was carried out on Sistan's native fenugreek (Trigonellafoenum_graecum). Colchicine concentration in four levels $\left(0.2,0.5,0.75\right.$ and $\left.1 \mathrm{gl}^{-1}\right)$ and the period of treatment in three levels $(6,12$ and 18 hours) were the two factors. The results showed that the concentration and period of colchicines' treatment and their interaction effects had significant role on auto tetra polyploidy levels. The highest level of polyploidy induction achieved by plunging seedling in concentration 0.5 gl- 1 of colchicine for 12 hours. Then, seeds were planted based on completely randomized block design with three replications and nineteen of morphological and physiological traits including plant height, number of leaf, length and wide of leaf, fresh and dry weight plant, chlorophyll a, chlorophyll b, total chlorophyll and chlorophyll c concentration were measured. The results showed that changed of ploidy levels from diploidy to tetraploidy by colchicine had significant effect for all morphological and physiological traits except fresh and dry weight plant.
\end{abstract}

Keywords: Auto tetra polyploidy, Colchicine, Fenugreek, Morphologic and physiologic trait 